\title{
Spin-Relaxation Anisotropy in a GaAs Quantum Dot
}

\author{
P. Scarlino, ${ }^{1}$ E. Kawakami, ${ }^{1}$ P. Stano, ${ }^{2,3}$ M. Shafiei,${ }^{1}$ C. Reichl ${ }^{4}$ W. Wegscheider, ${ }^{4}$ and L. M. K. Vandersypen ${ }^{1, *}$ \\ ${ }^{1}$ Kavli Institute of Nanoscience, TU Delft, Lorentzweg 1, 2628 CJ Delft, Netherlands \\ ${ }^{2}$ RIKEN Center for Emergent Matter Science, Wako-shi, Saitama 351-0198, Japan \\ ${ }^{3}$ Institute of Physics, Slovak Academy of Sciences, Dubravska cesta 9, 84511 Bratislava, Slovakia \\ ${ }^{4}$ Solid State Physics Laboratory, ETH Zurich, Schafmattstrasse 16, 8093 Zurich, Switzerland
}

(Received 2 September 2014; published 19 December 2014)

\begin{abstract}
We report that the electron spin-relaxation time $T_{1}$ in a GaAs quantum dot with a spin- $1 / 2$ ground state has a $180^{\circ}$ periodicity in the orientation of the in-plane magnetic field. This periodicity has been predicted for circular dots as being due to the interplay of Rashba and Dresselhaus spin orbit contributions. Different from this prediction, we find that the extrema in the $T_{1}$ do not occur when the magnetic field is along the [110] and [1 $1 \overline{1} 0]$ crystallographic directions. This deviation is attributed to an elliptical dot confining potential. The $T_{1}$ varies by more than 1 order of magnitude when rotating a $3 \mathrm{~T}$ field, reaching about $80 \mathrm{~ms}$ for the optimal angle. We infer from the data that in our device the signs of the Rashba and Dresselhaus constants are opposite.
\end{abstract}

The high control reached in the manipulation of a single electron spin in a semiconductor environment [1] is encouraging for future application of this natural two-level system for quantum computation technology. In GaAs, InAs, and other III-V quantum dots it has been shown that this manipulation can be realized using exclusively electrical fields [2,3]. Coupling of the electric field to the spins is mediated by the spin-orbit (SO) interaction naturally provided by the semiconductor host environment. The semiconductor environment also implies that the electron is intimately in contact with phonons, charge fluctuations, and nuclear spins, and these interactions are responsible for the relaxation and dephasing process of the electron spin.

During the last ten years, a significant experimental [4-10] and theoretical [11-15] effort has been devoted to understanding the effect of electron spin relaxation in lateral quantum dots (QDs). At magnetic fields of the order of Tesla, spin relaxation in GaAs dots was found to be dominated by the SO interaction in combination with piezoelectric phonons. Two contributions to the SO interaction usually dominate. The local electric field due to a crystal with bulk inversion asymmetry generates a Dresselhaus (D) SO contribution [16] which, for electrons confined in the plane (xy, with $x$ and $y$ along the [100] and [010] crystallographic directions, respectively) of the quantum well, can be written as $H_{D}=\beta\left(-\sigma_{x} P_{x}+\sigma_{y} P_{y}\right) / \hbar$, with $\hbar$ the Planck constant, $\beta$ the Dresselhaus SO coupling strength, $\mathbf{P}$ the electron kinematic momentum, and $\boldsymbol{\sigma}$ the vector of Pauli matrices. In addition, the electric field associated with the asymmetric confining potential along the heterostructure growth direction $(z$ along [001]) gives rise to the Rashba $(R)$ SO contribution [17], $H_{R}=\alpha\left(\sigma_{y} P_{x}-\sigma_{x} P_{y}\right) / \hbar$, with $\alpha$ the Rashba SO coupling strength. The effect of the SO interaction can be viewed as an effective magnetic field $\mathbf{B}_{\text {SO }}$ acting on the conduction electron spin, with an amplitude and direction that depend on the electron momentum [see Fig. 1(b)]. The interplay of $R$ and $D$ coupling gives rise to an anisotropy in the direction and magnitude of $\mathbf{B}_{\mathrm{SO}}$ in the plane of the quantum well. As a result, spin relaxation in a quantum dot is anisotropic in the direction of the inplane magnetic field $[13,14]$.

The anisotropy of electron spin relaxation originating from SO interaction has not been studied experimentally so far, even though it is highly relevant. Indeed, depending on the circumstances, it may be desirable to get long relaxation times or to make the relaxation process as fast as possible, for example, in order to rapidly initialize the spin [18]. The SO anisotropy similarly affects the strength of the effective
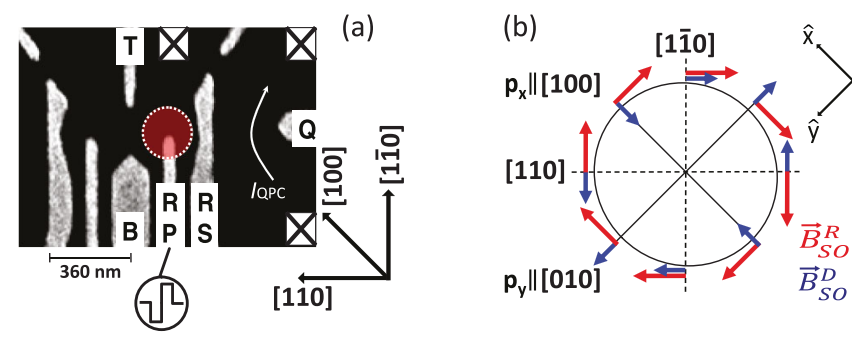

FIG. 1 (color). (a) Scanning electron micrograph of a device similar to the one measured. The black arrows indicate the crystallographic axes. The dotted red circle represents schematically the single QD position. (b) The spin-orbit field $\mathbf{B}_{\mathrm{SO}}$ acting on a conduction electron is shown by red and blue arrows (for a constant magnitude of $\mathbf{P}$ ), arising from the Rashba and Dresselhaus contributions (chosen to be different in modulus and $\alpha<0$, $\beta>0)$ and defined, respectively, as $\mathbf{B}_{\mathrm{SO}}^{R}=\left(\alpha / g \mu_{B}\right)\left(-P_{y}, P_{x}\right)$ and $\mathbf{B}_{\mathrm{SO}}^{D}=\left(\beta / g \mu_{B}\right)\left(-P_{x}, P_{y}\right)$, with $g$ the electron $g$ factor and $\mu_{B}$ the Bohr magneton. 
driving field for single-qubit rotations based on electric dipole spin resonance [19]. With a proper understanding, one can design future devices that optimally reconcile various requirements.

Here we present a measurement of $T_{1}$ as a function of the orientation of an in-plane magnetic field. We find a striking anisotropy with a $180^{\circ}$ periodicity, confirming the theoretical predictions experimentally. Comparison with the predictions indicates that also the dot shape anisotropy plays an important role. We discuss in detail what information is needed to determine the ratio of the Rashba and Dresselhaus coupling strengths in this case. We also provide guidance for sample design and magnetic field orientation in future experiments.

The experiment has been realized in a single depletion QD, see Fig. 1(a), created by applying a negative potential to surface gates on top of a $\mathrm{GaAs} / \mathrm{Al}_{0.33} \mathrm{Ga}_{0.67}$ As heterostructure, grown along the [001] direction. The $\mathrm{GaAs} / \mathrm{AlGaAs}$ interface is $85 \mathrm{~nm}$ deep, with Si-delta doping of about $1.3 \times 10^{12} \mathrm{~cm}^{-2}$ atoms $40 \mathrm{~nm}$ away from the 2DEG, and with carrier density and mobility of $1.2 \times 10^{11} \mathrm{~cm}^{-2}$ and $3.6 \times 10^{5} \mathrm{~cm}^{2} \mathrm{~V}^{-1} \mathrm{~s}^{-1}$, respectively (measured at $4 \mathrm{~K}$ ). The base temperature of the dilution refrigerator was $25 \mathrm{mK}$ and we estimated the electron temperature to be $130 \mathrm{mK}$ from transport measurements at zero magnetic field. From pulse spectroscopy measurements [20] we infer that the dot contains most likely three electrons (see Supplemental Material [21], Sec. I). Two electrons form a closed shell, with the third electron effectively acting as a spin- $1 / 2$ system. The orientation of the quantum dot gate pattern with respect to the main crystallographic directions is shown in Fig. 1(a). We applied a magnetic field in the 2DEG plane (at an angle $\phi$ with respect to the [100] direction) of modulus $3 \mathrm{~T}$, to ensure that the spin Zeeman energy $\left(\Delta_{z} \approx 76 \mu \mathrm{eV}\right.$, considering a $g$ factor of -0.44 [1]) is higher than the electron temperature $\left(k_{B} T_{\mathrm{el}} \approx 11 \mu \mathrm{eV}\right)$, as required for energy selective spin readout (see below) [5]. Real-time detection of the dot occupation is realized by monitoring the current through a quantum point contact (on the right side of the structure), amplified by a room temperature I-V converter, and low-pass filtered with a bandwidth of $30 \mathrm{kHz}$.

We measure the electron spin-relaxation time by applying a three- or four-stage pulse to gate RP [5] (see also Supplemental Material [21], Sec. I). The main observation is a striking variation in the relaxation time upon rotation of the in-plane magnetic field (Fig. 2). Figure 3 shows the measured relaxation time as a function of the magnetic field orientation over the whole $360^{\circ}$ range. The data show clearly the predicted $180^{\circ}$ periodicity and a remarkable variation in $T_{1}$ from 7 to $85 \mathrm{~ms}$ [Fig. 3(a)]. The maxima in $T_{1}$ are sharply peaked. When plotting the same data inverted, as $\Gamma=1 / T_{1}$ [Fig. 3(b)], we see a sinusoidal variation of the relaxation rate.

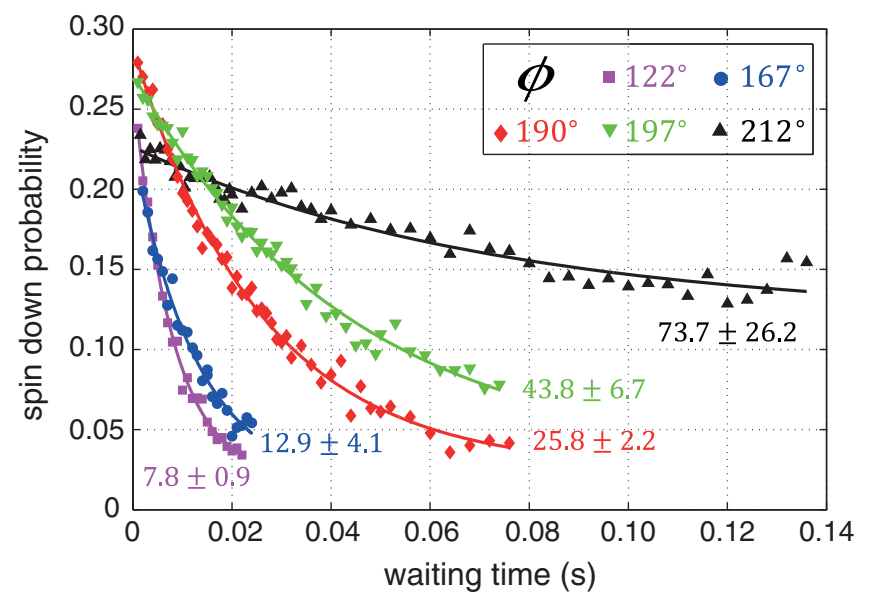

FIG. 2 (color). Measured spin-down probability (averaged over 5000 cycles) as a function of the waiting time between injection and readout (see Supplemental Material [21], Sec. I) for different angles $\phi$ of the $3 \mathrm{~T}$ in-plane magnetic field. The solid lines are fits to the data of the form $P_{\downarrow}=a \exp \left(-t / T_{1}\right)+b$. The fitted $T_{1}$ 's are indicated for each curve (in $\mathrm{ms}$ ). Small variations in $P_{\downarrow}(t=0)$ can arise from variations in the readout configuration in the course of the measurements. Measuring longer $T_{1}$ 's requires longer waiting times, with increased pulse distortion from the bias tee (see Supplemental Material [21], Sec. I), and therefore larger error.

To understand this sinusoidal modulation, it is useful to express the spin-relaxation rate in terms of a cross product of the external field $\mathbf{B}=B(\cos \xi \cos \phi, \cos \xi \sin \phi, \sin \xi)$, and the in-plane vector [24]

$$
\mathbf{n}=x\left(l_{D}^{-1},-l_{R}^{-1}, 0\right)+y\left(l_{R}^{-1},-l_{D}^{-1}, 0\right),
$$

which refers to crystallographic directions $\hat{x}=[100]$, and $\hat{y}=[010]$ through the operator of electron coordinates $\mathbf{r}=(x, y)$. The SO lengths, $l_{R, D}=\hbar^{2} /\left(2 m^{\star} \alpha, \beta\right)$, with $m^{\star}$ the effective electron mass, are defined as the distance traveled by an electron over which its spin is rotated by $\pi$ due to $\mathbf{B}_{\text {SO }}$ (typically 1-10 $\mu \mathrm{m}$ in GaAs). For a circular dot, the relaxation rate is (see Supplemental Material [21], Sec. II)

$$
\Gamma \propto\left|\mathbf{B} \times\left(l_{D}^{-1},-l_{R}^{-1}, 0\right)\right|^{2}+\left|\mathbf{B} \times\left(l_{R}^{-1},-l_{D}^{-1}, 0\right)\right|^{2},
$$

since the dipole operators $x$ and $y$ contribute equally [14]. Parametrizing the SO lengths by $l_{R}^{-1}=l_{\text {SO }}^{-1} \cos \vartheta$, and $l_{D}^{-1}=l_{\text {SO }}^{-1} \sin \vartheta$, a straightforward evaluation of Eq. (2) gives the known result $[13,14]$

$$
\Gamma \propto l_{\mathrm{SO}}^{-2}\left[\sin ^{2} \xi+\cos ^{2} \xi(1+\sin 2 \phi \sin 2 \vartheta)\right],
$$

which, for an in-plane magnetic field $(\xi=0)$ reduces to

$$
\Gamma \propto l_{\mathrm{SO}}^{-2}(1+\sin 2 \phi \sin 2 \vartheta) .
$$



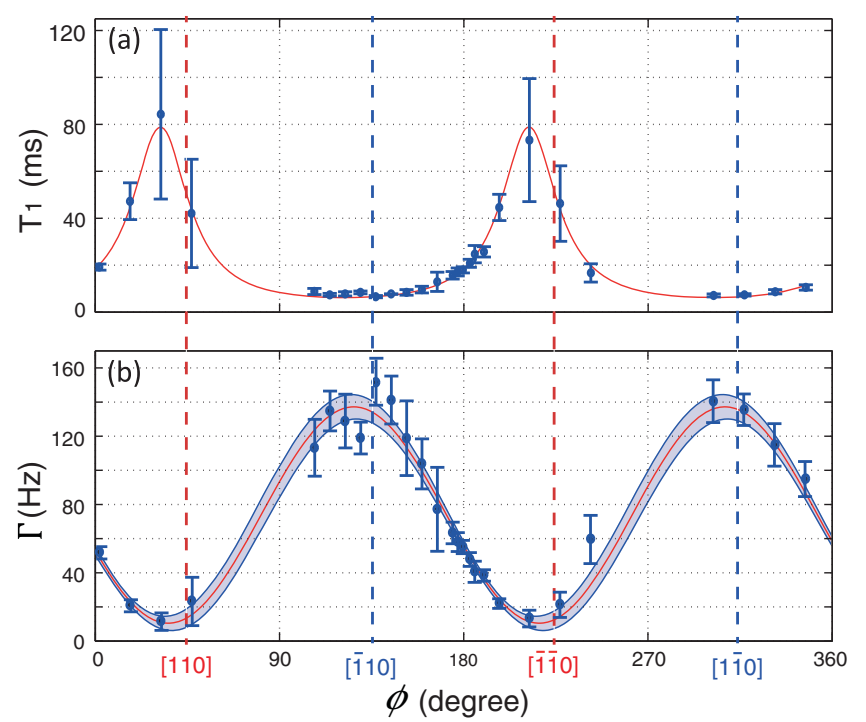

FIG. 3 (color). Angle dependence of the spin-relaxation time (a) and rate (b), which are separately extracted from exponential fits with either the relaxation rate or time in the exponent. The magnetic field is nearly in-plane, with $|\xi|<5^{\circ}$, while the in-plane angle $\phi$ has a systematic error of $\pm 3^{\circ}$. The red line is a fit to Eq. (7) with free parameters $\left(\phi_{\min }, \xi^{*}, b\right)$. The shaded region between the two blue curves indicates the $95 \%$ confidence interval. The dashed vertical lines show the positions of the extrema of $\Gamma$ predicted for a circular dot [at $\phi=45^{\circ}$ and $135^{\circ}$; see Eq. (3)].

For positive relative sign of the SO couplings, the rate reaches a maximum (minimum) with the external field along [110] ([1iㅣ]). If the relative sign is inverted, the positions of the minimum and maximum swap. If $R$ and $D$ have equal strength, the minimal rate is zero, while the sinusoidal modulation is reduced the more $R$ and $D$ differ in strength. Therefore, the relative strength of $R$ and $D$, including the relative sign, can be extracted from the dependence of $\Gamma$ on the magnetic field orientation.

Looking at the data in Fig. 3, the extrema of the rate are shifted by $\approx 10^{\circ}$ from the prediction of Eqs. (3) and (4). Similar offsets were observed in the dependence of SO induced avoided level crossings on the magnetic field orientation in InAs dots [25,26], and were explained by invoking anisotropic dot shapes [27]. The dot anisotropy influences also the spin-relaxation rate, as seen experimentally in Ref. [10] and anticipated theoretically in Ref. [12] considering the Dresselhaus coupling only. In addition to the observed shift, the dot in-plane elongation is indicated also by our spectroscopy data (see Supplemental Material [21], Sec. I): given the measured addition energy of about $3 \mathrm{meV}$, we would expect an orbital excitation energy of about $1 \mathrm{meV}$ [1], but in this sample, for the specific electrostatic configuration used for this experiment, the first orbital excitation energy is only $120 \mu \mathrm{eV}$. We will therefore assume that the dot is strongly anisotropic (elongated), with the confinement potential major axis rotated away from
[100] by an angle $\delta$. Neither this angle, nor the degree of anisotropy (nor any more details on the potential shape) are known.

To derive an analogue of Eq. (2) for an anisotropic dot, one should express Eq. (1) in coordinates $x^{\prime}, y^{\prime}$, rotated from the crystallographic axes by the angle $\delta$,

$$
\mathbf{n}=\mathbf{n}_{x^{\prime}} x^{\prime}+\mathbf{n}_{y^{\prime}} y^{\prime} .
$$

For an elongated dot, the excitations along the major axis $\left(x^{\prime}\right)$ dominate the transition matrix element (see Supplemental Material [21], Sec. II), and the rate is $[12,14,15]$

$$
\Gamma \propto\left|\mathbf{B} \times \mathbf{n}_{x^{\prime}}\right|^{2} .
$$

After some trigonometric manipulations, we are able to write the previous equation in the form

$$
\Gamma=b\left[\sin ^{2} \xi+\cos ^{2} \xi \sin ^{2}\left(\phi-\phi_{\min }\right)\right],
$$

where $b \equiv \kappa l_{\text {SO }}^{-2}(1+\sin 2 \delta \sin 2 \vartheta)$, with $\kappa$ a proportionality constant that sets the overall scale.

This expression predicts a rate varying sinusoidally upon in-plane rotation of the magnetic field, a feature in common with Eq. (3). However, the details of the dependence are very different. Here, unlike in Eq. (3), the optimal magnetic field angle for which the rate is minimal does depend on the ratio of Rashba and Dresselhaus coefficients (through $\vartheta$ ), and on the anisotropy axis of the dot (through $\delta$ ):

$$
\tan \phi_{\min }=-\frac{\cos (\delta-\vartheta)}{\sin (\delta+\vartheta)}
$$

To illustrate further the dependence of the relaxation rate on the orientation of the in-plane field and the dot major axis, we plot the prediction of Eq. (7) in Figs. 4(a) and 4(b) for different ratios of $l_{R}$ and $l_{D}$. When $l_{R}=-l_{D}$, the relaxation rate is minimal for $\phi=45^{\circ}$, regardless of the dot orientation, and also for $\delta=45^{\circ}$, regardless of the in-plane magnetic field orientation [Fig. 4(a)]. When $l_{R} \neq l_{D}$, the field orientation that minimizes the relaxation rate depends on the dot major axis orientation [Fig. 4(b)], with a $\pi$ periodicity.

We fit the data of Fig. 3(b) to Eq. (7) with $\phi_{\min }, \xi$, and $b$ as the fitting parameters. The fit is plotted in Fig. 3(b) as the red line. It agrees excellently with the data (fit goodness $R^{2} \approx 0.99$ ) and gives $\phi_{\min }=35.1^{\circ} \pm 1.1^{\circ}$, $\xi \approx 17.4^{\circ} \pm 1.1^{\circ}$, and $b=(139.2 \pm 3.5) \mathrm{s}^{-1}$. The fitted $17^{\circ}$ misalignment of the magnetic field out of the plane is, however, unrealistically large. We estimated it in our experimental setup via Shubnikov-de Haas oscillations, and can put an upper limit $|\xi|<5^{\circ}$ (see Supplemental Material [21], Sec. I). The unexpectedly high value of $\xi$ comes from the large value of the relaxation rate at its 

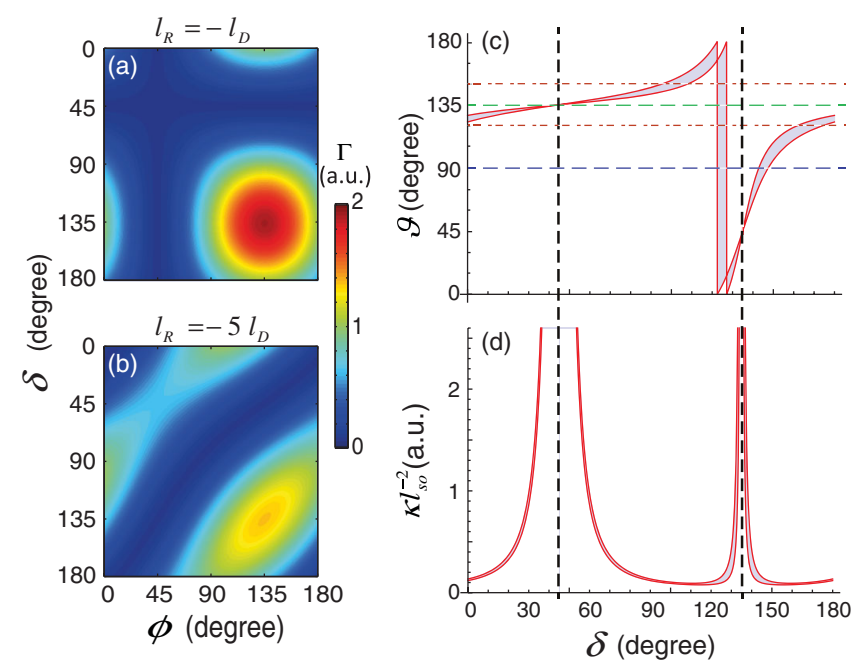

FIG. 4 (color). Calculated values of $\Gamma$ [Eq. (7)] as a function of the angle of the external magnetic field $\phi$ and the dot major axis $\delta$, for $\xi=0$ and (a) $l_{R} / l_{D}=-1$ and (b) $l_{R} / l_{D}=-5$. (c),(d) The result of the fit of the data of $\Gamma(\phi)$ from Fig. 3(b) to Eqs. (7) and (8), with $\vartheta$ and $\left(\kappa l_{\mathrm{SO}}^{-2}\right)$ (the latter in arbitrary units) as fit parameters, as a function of $\delta$. The shaded area between the two red curves indicates the $95 \%$ confidence interval (not taking into account the systematic error in $\phi$ ). The two black vertical lines indicate $\delta=45^{\circ}, 135^{\circ}$; the two red dotted lines and the green and blue horizontal lines are at $\vartheta=120^{\circ}, 150^{\circ}, 135^{\circ}$, and $90^{\circ}$, respectively.

minimum. We note, however, that this minimum value may also be dominated by other relaxation mechanisms that do not depend on $\phi$, such as the interaction with nuclear spins [12], or the contribution from the random part of the $R \mathrm{SO}$ coupling [28] which arises due to fluctuations in the dopant concentration in the $\delta$-doping layer. Contributions of orbital excitations along the minor axis also lead to a finite offset, as is suggested by Eq. (2). Without knowing more about the dot confinement shape, we did not find it reasonable to try to separate these possible contributions by introducing more fitting parameters. Instead, we relabel $\xi \rightarrow \xi^{*}$, reinterpreting it as an effective angle accounting for all these possibilities together.

Using the value of $\xi^{*}$ obtained from the fit and Eqs. (7) and (8), we can also perform a fit of the same data set with $\vartheta$ and $\kappa l_{\mathrm{SO}}^{-2}$ as free parameters, as a function of $\delta$. The fit results are plotted in Figs. 4(c) and 4(d). From there we conclude that without knowing the value of $\delta$, we cannot establish the relative strength of the $R$ and $D$ couplings, as all values of $\delta$ are possible, in principle. However, we can infer that, most probably, in our sample $\alpha$ and $\beta$ were of comparable magnitude and opposite sign $\left[120^{\circ} \leq\left(\vartheta=\arctan \left(l_{R} / l_{D}\right)\right) \leq 150^{\circ}\right]$, as these choices cover the larger portion of (a priori equally probable) values of $\delta$, in accordance of what is estimated in [29] for a similar heterostructure. There are two points, $\delta=45^{\circ}$ and $135^{\circ}$, where the rate $\kappa l_{\text {SO }}^{-2}$ diverges (see Supplemental Material [21], Sec. III). This indicates that such values of $\delta$ cannot be reconciled with our data. Indeed, as follows from Eq. (8), for these values $\phi_{\min }$ does not depend on the SO couplings and should be $45^{\circ}$ or $135^{\circ}$, which is different from what we measured. We furthermore note that if $\delta$ were known, $\alpha / \beta$ could be extracted directly. In order to also determine the absolute values of $\alpha$ and $\beta$, more information is needed, such as the energy level spectrum of the dot.

For future experiments, we give guidance for the optimal orientation of the quantum dot gate pattern and magnetic field relative to the crystal axes. First, since spin relaxation and electric dipole spin resonance (EDSR) based spin manipulation are governed by the same matrix elements for spin transitions, it is possible to simultaneously optimize for fast EDSR driven Rabi oscillations and for fast relaxation (useful for qubit reset [18]). In contrast, slow relaxation (useful for high-fidelity readout $[5,30]$ ) cannot be optimized together with fast EDSR, as long as the phonon coupling is isotropic, as then both the spinrelaxation rate and the EDSR rate scale with the same factor. In circular dots, this factor is given in Eq. (4). We see that the $R$ and $D$ terms maximally enhance or cancel each other when the external magnetic field is oriented along the [110] and [1 10$]$ crystallographic axes, as can be expected also from Fig. 1(b). Complete cancellation of the two contributions is possible only when $|\alpha|=|\beta|$. When $R$ and $D$ have very different strengths, $\Gamma$ does not vary with the magnetic field orientation. For anisotropic dots, the factor is given in Eq. (7). Here, $\Gamma$ oscillates with the field orientation and can reach zero (for $\xi=0$ ) regardless of the ratio of $\alpha$ and $\beta$. Finally, for maximizing the EDSR amplitude, in circular dots the external magnetic field has to point along [110] ([110]), if $\alpha \beta>0(\alpha \beta<0)$, and the driving electric field should be parallel to $\mathbf{B}$. In elongated dots the magnetic field should be oriented along the in-plane angle $\phi=\phi_{\min }+\pi / 2$, and the driving electric field should be along the dot soft axis. If the direction of the main dot

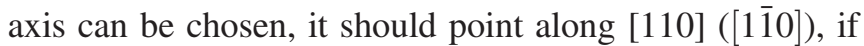
$\alpha \beta>0(\alpha \beta<0)$.

In conclusion, we show that the in-plane orientation of the magnetic field can strongly impact the spin-relaxation time in quantum dots. We observe a variation in $T_{1}$ by more than 1 order of magnitude when rotating the field in the 2DEG plane. We can take advantage of this dependence in future experiments to either maximize or minimize $T_{1}$. Furthermore, the dependence of $T_{1}$ on magnetic field angle provides a sensitive probe of the ratio of the $R$ and $D$ SO contributions, which can be used even when SO induced avoided level crossings are too small to be measured [31], which is the case of GaAs. What is needed is either a symmetric QD confining potential or, for an elliptical dot, a good estimate of the magnitude and direction of the QD anisotropy. Similar considerations are also valid for singlettriplet qubits [32] [33], where the easy axis is given by the double dot dipole axis [31]. 
We acknowledge K.C. Nowack for fabricating the sample, V. Golovach for useful discussions, and R. Schouten for technical support. Research was supported by the Intelligence Advanced Research Projects Activity through the Army Research Office Grant No. W911NF-121-0354, the European Research Council, the Dutch Foundation for Fundamental Research on Matter, and the Swiss National Science Foundation.

*Corresponding author.

L.M.K.Vandersypen@tudelft.nl

[1] R. Hanson, J. R. Petta, S. Tarucha, and L. M. K. Vandersypen, Rev. Mod. Phys. 79, 1217 (2007).

[2] K. C. Nowack, F. H. L. Koppens, Y. V. Nazarov, and L. M. K. Vandersypen, Science 318, 1430 (2007).

[3] S. Nadj-Perge, S. M. Frolov, E. P. A. M. Bakkers, and L. P. Kouwenhoven, Nature (London) 468, 1084 (2010).

[4] T. Fujisawa, D. G. Austing, Y. Tokura, Y. Hirayama, and S. Tarucha, Nature (London) 419, 278 (2002).

[5] J. M. Elzerman, R. Hanson, L. H. Willems van Beveren, B. Witkamp, L. M. K. Vandersypen, and L. P. Kouwenhoven, Nature (London) 430, 431 (2004).

[6] M. Kroutvar, Y. Ducommun, D. Heiss, M. Bichler, D. Schuh, G. Abstreiter, and J. J. Finley, Nature (London) 432, 81 (2004)

[7] A. C. Johnson, J. R. Petta, J. M. Taylor, A. Yacoby, M. D. Lukin, C. M. Marcus, M. P. Hanson, and A. C. Gossard, Nature (London) 435, 925 (2005).

[8] S. Amasha, K. MacLean, I. P. Radu, D. M. Zumbuhl, M. A. Kastner, M. P. Hanson, and A. C. Gossard, arXiv:cond-mat/ 0607110

[9] T. Meunier, I. T. Vink, L. H. Willems van Beveren, K. J. Tielrooij, R. Hanson, F. H. L. Koppens, H. P. Tranitz, W. Wegscheider, L. P. Kouwenhoven, and L. M. K. Vandersypen, Phys. Rev. Lett. 98, 126601 (2007).

[10] S. Amasha, K. MacLean, I. P. Radu, D. M. Zumbühl, M. A. Kastner, M. P. Hanson, and A. C. Gossard, Phys. Rev. Lett. 100, 046803 (2008).

[11] A. V. Khaetskii and Y. V. Nazarov, Phys. Rev. B 61, 12639 (2000).

[12] A. V. Khaetskii and Y. V. Nazarov, Phys. Rev. B 64, 125316 (2001).

[13] V. N. Golovach, A. Khaetskii, and D. Loss, Phys. Rev. Lett. 93, 016601 (2004).

[14] P. Stano and J. Fabian, Phys. Rev. B 74, 045320 (2006).

[15] P. Stano and J. Fabian, Phys. Rev. Lett. 96, 186602 (2006).
[16] G. Dresselhaus, Phys. Rev. 100, 580 (1955).

[17] Y. A. Bychkov and E. I. Rashba, J. Phys. C 17, 6039 (1984).

[18] V. Srinivasa, K. C. Nowack, M. Shafiei, L. M. K. Vandersypen, and J. M. Taylor, Phys. Rev. Lett. 110, 196803 (2013).

[19] V. N. Golovach, M. Borhani, and D. Loss, Phys. Rev. B 74, 165319 (2006).

[20] J. M. Elzerman, R. Hanson, L. H. Willems van Beveren, L. M. K. Vandersypen, and L. P. Kouwenhoven, Appl. Phys. Lett. 84, 4617 (2004).

[21] See Supplemental Material at http://link.aps.org/ supplemental/10.1103/PhysRevLett.113.256802, which includes Refs. [22,23], for further experimental details, a derivation of Eqs. (2) and (6) in the main text and a discussion of the divergences in the fitting parameter $\kappa l_{\mathrm{SO}}^{-2}$.

[22] M. Raith, P. Stano, and J. Fabian, Phys. Rev. B 86, 205321 (2012).

[23] F. Baruffa, P. Stano, and J. Fabian, Phys. Rev. Lett. 104, 126401 (2010).

[24] L. S. Levitov and E. I. Rashba, Phys. Rev. B 67, 115324 (2003)

[25] S. Takahashi, R. S. Deacon, K. Yoshida, A. Oiwa, K. Shibata, K. Hirakawa, Y. Tokura, and S. Tarucha, Phys. Rev. Lett. 104, 246801 (2010).

[26] Y. Kanai, R. S. Deacon, S. Takahashi, A. Oiwa, K. Yoshida, K. Shibata, K. Hirakawa, Y. Tokura, and S. Tarucha, Nat. Nanotechnol. 6, 511 (2011).

[27] M. P. Nowak, B. Szafran, F. M. Peeters, B. Partoens, and W. J. Pasek, Phys. Rev. B 83, 245324 (2011).

[28] E. Y. Sherman and D. J. Lockwood, Phys. Rev. B 72, 125340 (2005).

[29] W. W. Yu, S. M. Frolov, S. Luescher, J. A. Folk, and W. Wegscheider, arXiv:1009.5702.

[30] K. C. Nowack, M. Shafiei, M. Laforest, G. E. D. K. Prawiroatmodjo, L. R. Schreiber, C. Reichl, W. Wegscheider, and L. M. K. Vandersypen, Science 333, 1269 (2011).

[31] V. N. Golovach, A. Khaetskii, and D. Loss, Phys. Rev. B 77, 045328 (2008).

[32] V. Kornich, C. Kloeffel, and D. Loss, Phys. Rev. B 89, 085410 (2014).

[33] According to our analysis (not reported here), the dipole matrix elements between a singlet [either $(1,1)$ and $(0,2)$ ] and a polarized triplet $(1,1)$ in a biased two electron double dot tuned close to a singlet(1,1)-singlet $(0,2)$ anticrossing have the same angular dependence as the single electron matrix elements discussed here. 